\title{
Contribución a la caracterización de los espacios agrarios castreños: documentación y análisis palinológico de una posible terraza de cultivo en el castro de Follente (Caldas de Reis, Pontevedra)
}

\begin{abstract}
A contribution to the characterisation of Iron Age agrarian spaces: documentation and pollen analysis of a possible cultivation terrace in the castro of Follente (Caldas de Reis, Pontevedra)
\end{abstract}

José Antonio López Sáez (*)

Lourdes López Merino (*)

Sebastián Pérez Díaz $(*)$

César Parcero-Oubiña $(* *)$

Felipe Criado-Boado (**)

\section{RESUMEN}

Se presentan los resultados de una caracterización palinológica realizada en un perfil en el cual se ha identificado una probable estructura de cultivo artificial (terraza) de finales de la Edad del Hierro, asociada a un castro en Galicia. El análisis combinado de las evidencias estratigráficas y palinológicas permite proponer una secuencia de transformación del paisaje en la que, de forma poco esperable, la mayor intensificación en el uso de la zona, correspondiente a la ocupación del castro y el desarrollo de estructuras agrarias, coincide con un episodio de incremento de los espacios forestales en el entorno. Sin embargo, el análisis realizado permite documentar la progresiva construcción de un paisaje cada vez más antropizado y donde los efectos de la acción humana se van haciendo más visibles.

\footnotetext{
ABSTRACT

A series of samples extracted from the stratigraphic profile of a possible cultivation terrace located in the sur-

(*) Grupo de Investigación Arqueobiología. Instituto de Historia. Centro de Ciencias Humanas y Sociales. CSIC. Albasanz 26-28. 28037 Madrid. Correos electrónicos: joseantonio.lopez@cchs.csic.es lourdes.lopezmerino@cchs.csic.es sebastian.perez@cchs.csic.es

(**) Laboratorio de Patrimonio ( $\mathrm{LaPa})$; Consejo Superior de Investigaciones Científicas (CSIC). Instituto de Estudos Galegos Padre Sarmiento. San Roque 2. 15704 Santiago de Compostela. Correos electrónicos:

cesar.parcero-oubina@iegps.csic.es

felipe.criado-boado@iegps.csic.es

Recibido: 12-I-2009; aceptado: 12-VI-2009.
}

roundings of an Iron Age hillfort in Galicia were used for pollen analysis. The combined results of both the stratigraphic information and the pollen analysis allow the proposal of a sequence of landscape transformation where, rather unexpectedly, the greater intensification in the use of the area, corresponding to the occupation of the hillfort and the construction of cultivation structures, coincides with an increase in the forested areas in the environment. However, the results of the analysis also document the construction of a landscape in which the effects of human action are progressively more visible.

Palabras clave: Arqueología del Paisaje; Análisis polínico; Edad del Hierro; Castros; Estructuras agrarias; Terrazas; Holoceno.

Key words: Landscape Archaeology; Pollen analysis; Iron Age; Hillforts; Agrarian structures; Terraces; Holocene.

\section{INTRODUCCIÓN}

El análisis de las formas de los espacios agrarios es una de las líneas más exploradas dentro de algunas corrientes de la arqueología del paisaje, especialmente las desarrolladas en Francia o Italia (p.e. Chouquer y Favory 1991; Guilaine (ed.) 1991, entre otros muchos). Para el caso de la Península Ibérica, hay también una ya larga tradición centrada en las formas de los paisajes de 
épocas romana y medieval, pero son escasas las experiencias relativas a paisajes agrarios anteriores (afortunadamente, cada vez menos escasas, un buen ejemplo es Orejas (coord.) 2006).

Para el caso de la Edad del Hierro del noroeste, hace unos años el desarrollo de actuaciones de seguimiento y control de obras públicas en Galicia posibilitó documentar de forma directa una serie de ejemplos puntuales de posibles estructuras agrarias en la periferia o proximidad de algunos castros. La lectura arqueológica de este conjunto de evidencias permitió una publicación de las mismas a partir de su interpretación estratigráfica (Parcero-Oubiña 1999) y de la combinación de ésta con un análisis contextual de su localización (Parcero-Oubiña 2006).

En este trabajo aportamos un nuevo nivel de significación, que viene dado por la disponibilidad de resultados de analíticas realizadas sobre muestras tomadas en algunas de estas estructuras, y que permiten ofrecer una caracterización más detallada de las mismas desde una perspectiva paleoambiental hasta ahora no explorada.

\section{CASO DE TRABAJO: CASTRO DE FOLLENTE (CALDAS DE REIS, PONTEVEDRA)}

El castro de Follente se localiza cerca del núcleo de Caldas de Reis, en las estribaciones finales de Monte Xiabre, en un pequeño outeiro que destaca sobre las tierras bajas del valle del Umia (Fig. 1). Es un castro relativamente extenso para lo habitual en este área, aunque dentro de valores medios: tomando la totalidad de sus estructuras visibles e incluyendo las identificadas en su periferia, ocupa aproximadamente unas 3,2 Has (Parcero-Oubiña 2006). Su estructura es relativamente compleja (Fig. 2).

Para lo que nos ocupa ahora, lo más destacable es la sucesión de una serie de niveles aterrazados hacia el sur, ya fuera del perímetro de lo que pueden considerarse las estructuras defensivas visibles del poblado; algunos de ellos podrían ser naturales, con afloramientos visibles, pero otros son claramente artificiales. Resulta difícil identificar con precisión la fisonomía completa de este espacio, ya que la zona ha sido sometida a importantes cambios recientes, como la apertura de pistas forestales, la construcción de la autopista A-9 al oeste o, especialmente, los cambios en los usos

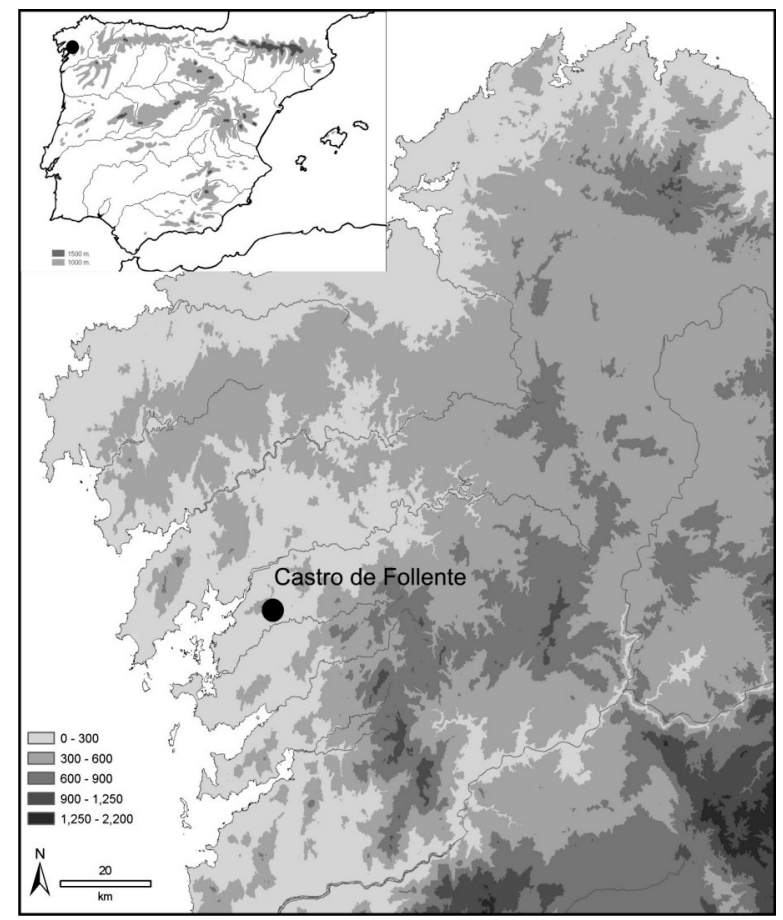

Fig. 1. Localización del castro de Follente.

del suelo y la intensa repoblación forestal. Esto ha generado un paisaje "disfrazado" en el que, sin embargo, ha sido posible documentar trazas evi-

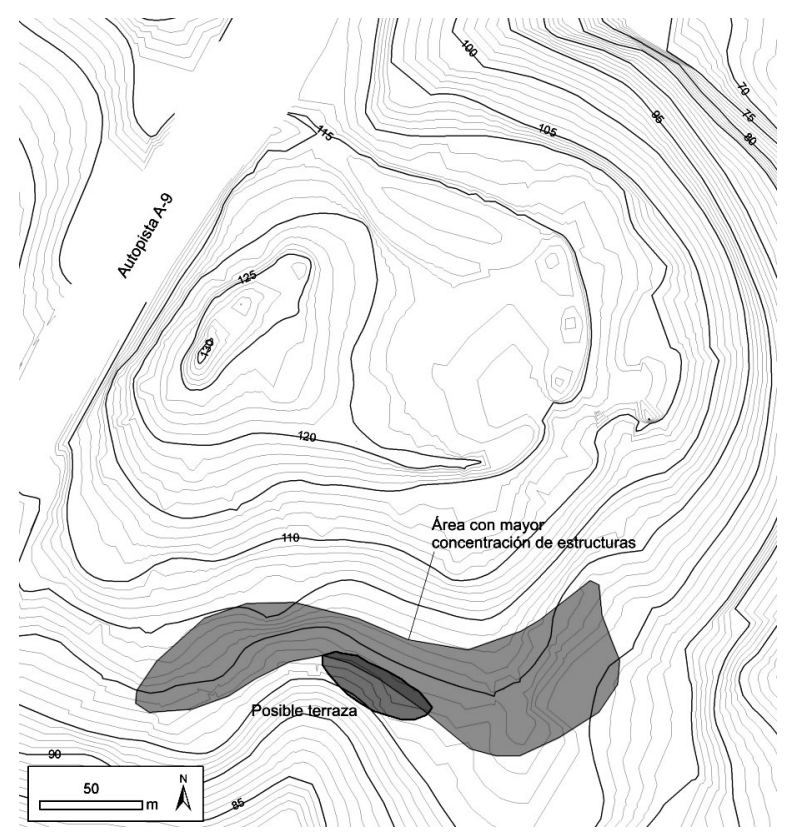

Fig. 2. Planimetría del castro de Follente con la localización de la posible estructura de cultivo. 


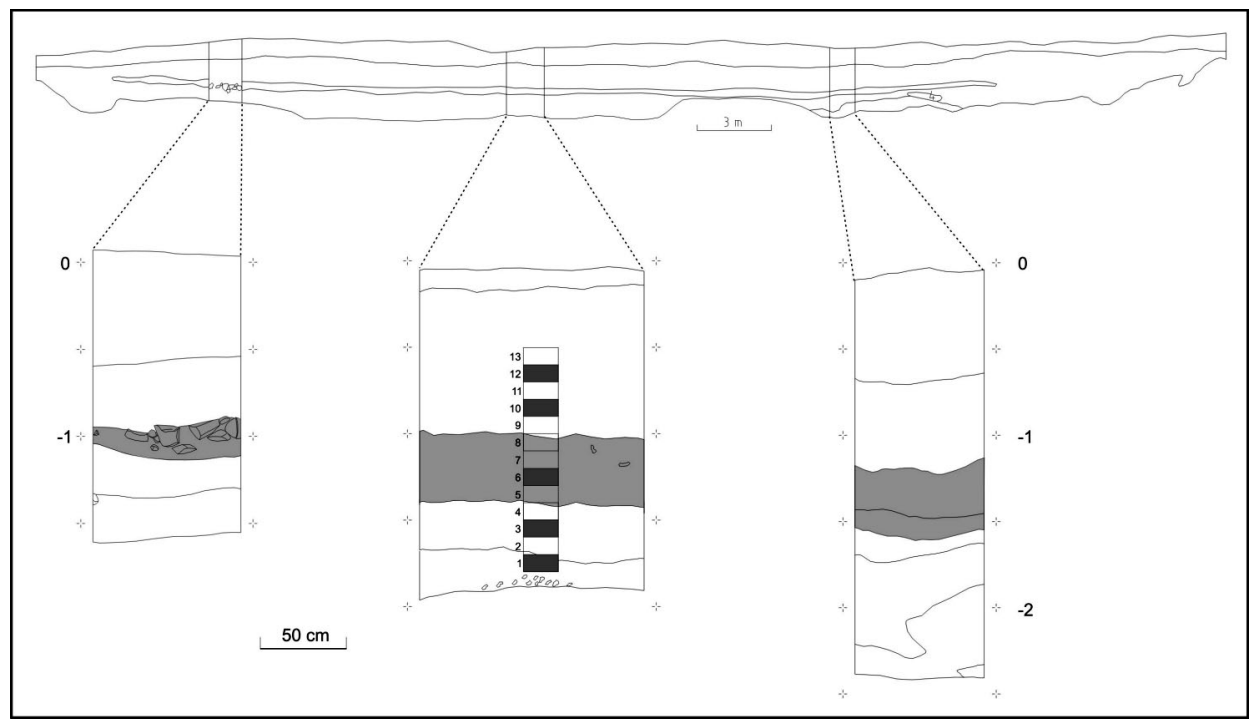

Fig. 3. Perfil de la posible estructura agraria al sur del castro de Follente con indicación de la columna polínica analizada.

dentes de un espacio agrario complejo, fuertemente artificializado y que, sin duda, debió destinarse a un laboreo intensivo.

La construcción de la zanja del gasoducto Valga-Tui por las inmediaciones de este castro se remonta a 1997, fruto de la secuencia de decisiones derivadas de la necesidad de desviar el proyecto inicial, que lo atravesaba por su parte central (más detalles en Parcero-Oubiña 1999). Como resultado de este cambio, se evitó la afección sobre las estructuras visibles del sitio, pero se puso al descubierto una gran cantidad de elementos enterrados, casi todos ellos curiosamente en el área del trazado más alejada del castro, al sur y sureste: estructuras lineales, fosas, fosos y dos elementos posiblemente relacionados con prácticas agrarias. El conjunto de estructuras documentadas permite dibujar una imagen del poblado más extensa y compleja que la sugerida por sus estructuras defensivas, pues se han podido reconstruir una serie de espacios periféricos a lo que habría sido el área de habitación del poblado (aquella delimitada por las murallas y fosos visibles en superficie). Estos espacios incluyen al menos varios fosos y zanjas de delimitación adicionales y un espacio probablemente agrario al sur, compuesto por al menos un par de terrazas de cultivo y restos de un camino encajado que daría servicio a este sector (el registro detallado de estas estructuras y una propuesta de reconstrucción se encuentran en Parcero-Oubiña 1999, 2006).
Una de las estructuras de ese espacio al sur es la que nos interesa ahora (1). Se localiza en la zona en la que el trazado atraviesa una pequeña vaguada ocupada por una sucesión de terrazas de cultivo hoy abandonadas y replantadas con eucaliptos. La estructura se define como una amplia zona de colmatación que se extiende por los perfiles de la zanja del gasoducto a lo largo de unos $50 \mathrm{~m}$ lineales. La profundidad media supera los $2 \mathrm{~m}$, contando la totalidad del perfil. Mediante un trabajo de limpieza manual de estos perfiles se pudo identificar y documentar gráficamente una secuencia estratigráfica uniforme a lo largo de esos 50 m (Fig. 3). Esta secuencia está integrada por tres episodios esenciales.

En la parte superior del perfil se localiza una profunda acumulación de, al menos, dos sedimentos, muy semejantes, ambos de tierra de grano grueso, con gravas, sin agregación ni estructura de suelo, que agrupamos como parte de un mismo proceso de colmatación. En ellos no se documentó material arqueológico alguno. Su origen parece relativamente reciente, y podría relacionarse con la deposición de amplias capas de tierra fruto de la intensificación del laboreo en una zona que debió estar dedicada al cultivo intensivo.

(1) Para una descripción detallada del asentamiento y del conjunto de estructuras documentadas en su entorno, ver Parcero-Oubiña 1999. 
Esta acumulación cubre un segundo episodio compuesto por una estrecha banda de tierra más agregada, de menos de $50 \mathrm{~cm}$ de grosor, compuesta por material de grano fino pero sin estructura de suelo. Contiene pequeños fragmentos de cerámica castreña, todos ellos rodados y que no nos permiten ser más concretos en su adscripción. Hacia la parte superior aparecen algunas piedras. En el extremo Este de la estructura, este nivel se prolonga en una estrecha y homogénea línea de escombro que se compone sobre todo de piedras, tierra arcillosa y abundantes fragmentos de cerámica, esta vez sin rodar y muchos de los cuales unen entre sí. Parece claro que se trata de un derrumbe de algún tipo de construcción.

Este episodio intermedio es el más interesante. En primer lugar porque contiene gran cantidad de material castreño, lo cual indica que se trata de un depósito antiguo, coetáneo con la ocupación del poblado. Su posible lectura, a partir del escaso grosor y poca agregación del material edáfico que lo compone, es que se trate de un antiguo suelo de cultivo al que el material cerámico ha llegado como parte de un proceso de abonado (la cerámica aparece muy fragmentada y rodada). La ausencia de estructuras claras, la fragmentación y rodamiento del material y los rasgos edafológicos del sedimento, que no es un suelo natural, apuntan a su posible origen como parte de un proceso de cultivo.

Este suelo de cultivo se habría desarrollado sobre un episodio inferior que sí constituye un paleosuelo natural, preexistente a toda la secuencia que se desarrolla por encima. Este paleosuelo está compuesto por dos horizontes superiores (A1 y A2), no demasiado profundos, que dan paso directamente a la roca base descompuesta (saprolita). Contienen muy poco material arqueológico, siempre situado en la parte superior del horizonte más elevado (A1), justo en contacto con el horizonte central de la secuencia.

\section{ANÁLISIS PALINOLÓGICO}

Como forma de discernir el paleopaisaje y los rasgos paleoeconómicos que pueden inferirse del posible paleosuelo castreño, así como de la información paleoambiental del resto de niveles estudiados, se procedió a realizar un análisis polínico sobre una columna de muestras tomada en el perfil que acabamos de describir. La columna consta de 13 muestras, numeradas de techo (13) a base (1) respectivamente: las muestras 13 a 9 proceden del episodio superior, las 8 a 5 del posible suelo de cultivo castreño o episodio central y las 4 a 1 del episodio inferior.

Todas las muestras (10 g) fueron tratadas químicamente utilizando la metodología clásica para yacimientos arqueológicos propuesta por Girard y Renault-Miskovsky (1969), con concentración del polen mediante flotación en licor denso de Thoulet, adaptando las modificaciones expuestas por Goeury y Beaulieu (1979). La determinación de los tipos polínicos se realizó básicamente de acuerdo a Moore et al. (1991), y Reille (1992, 1995), así como mediante la comparación con la colección de referencia del Laboratorio de Arqueobiología del Instituto de Historia (CSIC, Madrid). Los microfósiles no polínicos fueron identificados de acuerdo a López Sáez et al. (1998, 2000).

En la figura 4 queda recogido el diagrama palinológico correspondiente al análisis realizado. El espectro polínico de cada muestra ha sido establecido a partir de la contabilización como término medio de alrededor de 150 pólenes, que constituye la suma base polínica (SBP). Los valores relativos de los taxones, tanto arbóreos como no arbóreos, se han obtenido a partir de la suma de los valores absolutos de cada uno de ellos y vienen referidos a la suma base polínica. De ella se han excluido los pólenes de especies hidro-higrófitas, los microfósiles no polínicos, así como ciertas zoófilas (Cichorioideae, Cardueae) de acuerdo a su hipotética sobrerrepresentación (Bottema 1975; Carrión 1992).

Del episodio inferior (muestras 4 a 1) únicamente las muestras 1 y 3 fueron fértiles en contenido polínico. Las muestras 2 y 4 son completamente estériles, aunque en ambas se detectó un altísimo contenido en microcarbones cuyo origen es difícil de precisar. Es probable que la esterilidad de estas muestras obedezca a algún hecho relacionado con eventos de incendio. De hecho, en ambas muestras fértiles se han identificado ascosporas fúngicas de Chaetomium sp, un microfósil no polínico de ecología carbonícola y, por lo tanto, indicador de procesos de incendio que, en el caso que nos concierne, posiblemente sean de origen antrópico, ya que, como más tarde se detalla, su presencia se relaciona con la documentación en paralelo de indicadores polínicos de antropización y un medio ambiente no demasiado forestado (López Sáez et al. 1998, 2000). 


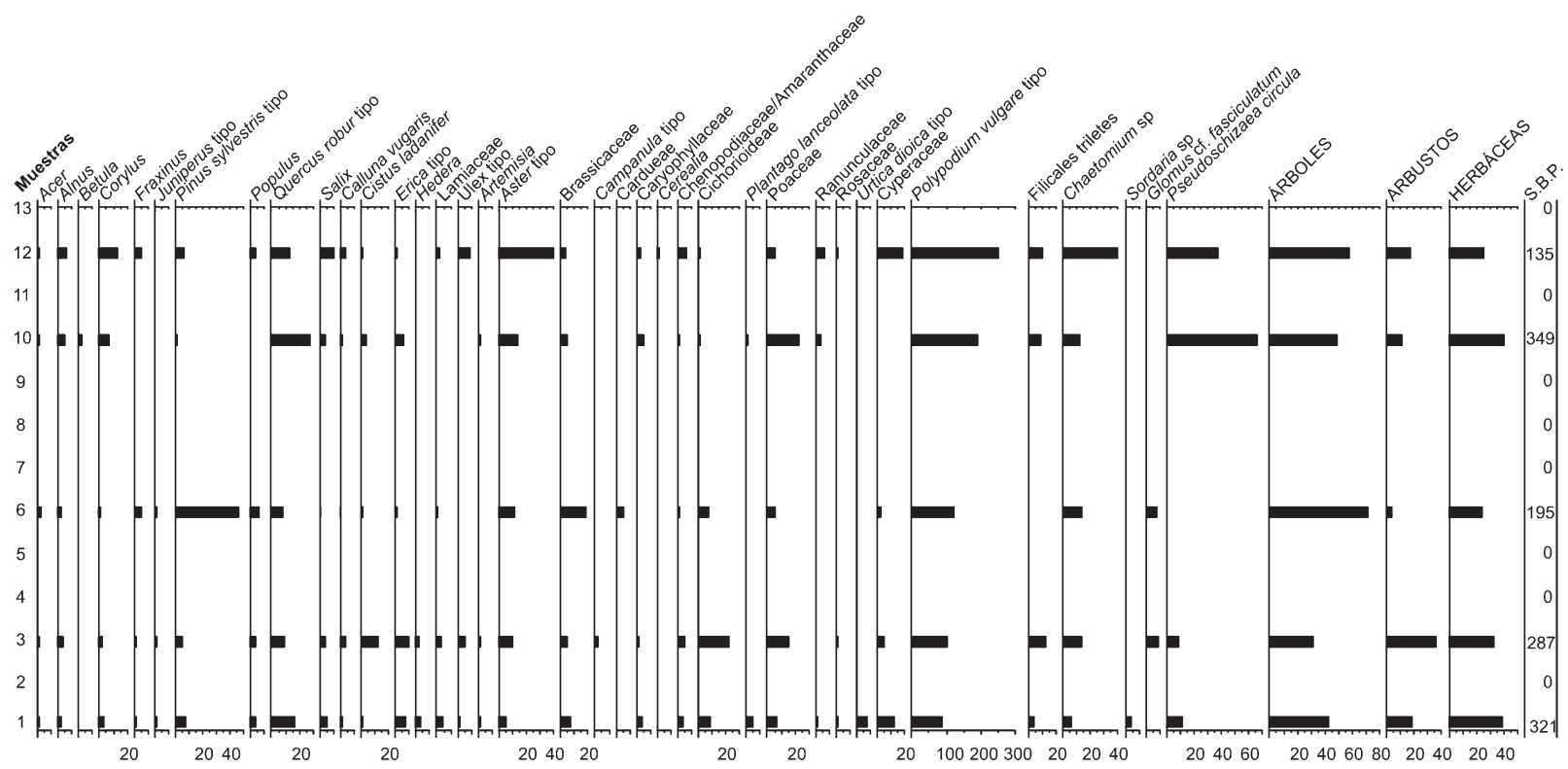

Fig. 4. Diagrama palinológico del posible espacio agrario del castro de Follente.

Las muestras 1 y 3 dan cuenta de un paisaje relativamente abierto, donde el porcentaje de árboles no supera el $40 \%$, siendo los carballos (Quercus robur tipo) el palinomorfo arbóreo mejor representado con un 10-20\%, y otros elementos arbóreos menores el aliso (Alnus), arce (Acer), avellano (Corylus), fresno (Fraxinus), chopo (Populus), sauce (Salix) y pino (Pinus sylvestris tipo). La flora arbustiva tampoco es muy importante, reflejando posiblemente esa situación de bosque abierto, ya que alcanza porcentajes de ca. $20 \%$ en la muestra 1 y ca. $40 \%$ en la 3. Entre sus elementos más importantes se encuentran jaras (Cistus ladanifer), tojos (Ulex tipo) y brezos (Erica tipo).

La flora herbácea en los espectros polínicos de las dos muestras del episodio inferior tiene porcentajes no lejanos de los árboles, siendo sus palinomorfos más representativos las gramíneas (Poaceae), así como otros elementos característicos de ambientes ruderalizados o, en general, antropizados (Behre 1981) caso de Aster tipo, Cichorioideae, Plantago lanceolata tipo, Urtica dioica tipo, etc. Al menos estos dos últimos palinomorfos, en referencia a la muestra 1, podrían hacernos suponer cierto tipo de presión pastoral pues son característicos de tales actividades, y de hecho aparecen paralelamente a ascosporas coprófilas de Sordaria sp., un microfósil no políni- co indicador de actividad ganadera in situ (López Sáez et al. 2000).

En resumen, el paleopaisaje inferido durante el episodio inferior correspondería al de un bosque de quercíneas caducifolias (carballos) relativamente abierto por procesos de incendios antrópicos, acompañado en todo caso de un rico elenco de especies arbóreas mesófilas (alisos, avellanos, fresnos, sauces, arces, chopos, etc.), de pino posiblemente de carácter alóctono, con un dosel arbustivo indicativo de un amplio desarrollo de etapas degradativas seriales del bosque climácico y, finalmente, de una cobertura herbácea prolija en elementos indicadores de ambientes antropizados, que en la muestra basal 1 indicarían además cierta presión de tipo pastoral.

Del episodio central, el correspondiente al posible suelo de cultivo castreño, desafortunadamente sólo una muestra fue fértil a nivel polínico, la 6 , mientras que las otras tres $(5,7$ y 8$)$ resultaron estériles. Al igual que antes, la esterilidad de tales muestras va ligada a una altísima concentración de microcarbones en todas ellas, por lo que pueden aceptarse las mismas consideraciones antes expuestas, con la consiguiente aparición de Chaetomium sp. y de otros indicadores polínicos de antropización referidos.

La muestra 6, a diferencia de lo que ocurría en el episodio inferior, da cuenta ahora de un impor- 
tante desarrollo forestal, con porcentajes de árboles que alcanzan casi el $80 \%$. No obstante, estos datos tenemos que tomarlos con mucha cautela, pues en realidad tal desarrollo arbóreo obedece exclusivamente al progreso de los pinos que aquí superan el $40 \%$, mientras que el resto de palinomorfos arbóreos permanecen más o menos estables salvo el carballo que ve disminuir ligeramente sus porcentajes. Estos datos nos permitirían suponer cierta degradación del bosque climácico local, el carballal, y el desarrollo de pinares en zonas alejadas de la nuestra aunque en cualquier caso de una manera relativamente importante, pues el porcentaje de polen de pino es elevado, lo cual se explica fácilmente teniendo en cuenta el carácter anemófilo y la gran producción polínica de este taxón. No obstante, dado que sólo tenemos una muestra para este episodio, mayor precisión no es posible a tal respecto.

La flora arbustiva se reduce igualmente, según denotan los porcentajes de brezos, jaras y tojos (que desaparecen), demostrando que la citada deforestación del carballal habría incluso alcanzado a las formaciones seriales propias de las etapas degradativas de éste, es decir a los brezales, jarales y tojales.

Entre la flora herbácea se siguen confirmando los mismos elementos antes citados indicativos de paisajes antrópicos, de ambientes ruderalizados, a los que podría añadirse ahora un máximo para Brassicaceae y Cardueae. A diferencia del episodio inferior, ahora no encontramos indicio alguno de presión pastoral.

En resumen, durante el episodio central el bosque climácico de la zona, el carballal, se habría deforestado aún más, lo mismo que la cobertura arbustiva acompañante, como consecuencia de una antropización continuada, especialmente mediada por la incidencia del fuego, sin que ahora tengamos constancia de actividades pastoriles.

Finalmente, del episodio superior han sido tres las muestras estériles $(9,11$ y 13$)$ y dos las fértiles $(10$ y 12$)$. Aunque puedan seguir sosteniéndose las causas antes descritas de esterilidad, la existencia ahora de importantes niveles de sedimentos de grano grueso, asociados a grava, podría implicar una esterilidad polínica por fenómenos de abrasión (López Sáez et al. 2003). En este episodio superior el bosque caducifolio se recupera, especialmente el carballo que llega a superar en la muestra 10 el $20 \%$ y tiene ca. $10 \%$ en la 12. De la misma manera el avellano y el sauce igualmente prosperan, al igual que el aliso y la primera aparición del abedul, mientras que los pinos se reducen drásticamente.

El porcentaje de arbustivas es bajo, no superior al $20 \%$, siendo importante ahora el desarrollo del tojo que alcanza el $8 \%$, y teniendo en cuenta su carácter zoófilo estos porcentajes indicarían fuerte incidencia local.

Entre las herbáceas, no obstante, se siguen confirmando procesos antrópicos y palinomorfos indicativos de antropización semejantes a los citados con anterioridad, incluyendo ahora la única documentación en el palinograma de polen de cereal, en todo caso con porcentaje inferior al $3 \%$, que no nos permitiría confirmar su cultivo local aunque sí probablemente en un área no muy lejana a la zona de estudio (López Sáez y López Merino 2005). La abundancia ahora de Cyperaceae podría obedecer a una humedad ambiental y edáfica más elevada, que explicaría el desarrollo de estos pastos húmedos así como del amplio elenco de elementos arbóreos antes descrito, especialmente reseñable en el caso de especies versadas hacia el agua como aliso, abedul o sauce.

No hay indicios de pastoralismo. La fuerte presencia en este episodio de Pseudoschizaea circula podría estar vinculada a procesos erosivos relacionados bien con el aumento de humedad bien con las actividades antrópicas (López Sáez et al. 2000).

\section{DISCUSIÓN Y CONCLUSIONES}

El análisis de la columna polínica realizada sobre el perfil de la estructura ha permitido obtener evidencias complementarias no sólo para caracterizarla, sino especialmente para reconocer una serie de transformaciones en el paisaje vegetal del área de Follente. Las posibles lecturas derivadas de la combinación de estos resultados con los trabajos anteriores de caracterización formal y estratigráfica de esta estructura (y del conjunto de otras evidencias documentadas en la zona) (Parcero-Oubiña 1999, 2006), encuentran dos importantes limitaciones que conviene señalar.

La primera es el elevado número de muestras cuyo contenido polínico ha sido estéril, lo que hace que la secuencia que podemos presentar se reduzca a sólo 5 muestras; esta cuestión es todavía más acusada en el caso del episodio estratigráfico más interesante de la secuencia, el cen- 
tral, en el que sólo una de las muestras analizadas ha aportado información polínica. La segunda limitación viene dada por la ausencia de dataciones absolutas que se puedan vincular con la secuencia estratigráfica de la estructura. A partir de la documentación de materiales cerámicos en el episodio central hemos podido proponer con mucha confianza su adscripción a una Edad del Hierro probablemente tardía, tal vez incluso ya en época romana. Esto es así no sólo por el material documentado en esta estructura concreta, que aparece, como apuntamos, bastante fragmentado y rodado, como por el conjunto de materiales localizados en todo el entorno del castro, y sobre el que volveremos más adelante. Sin embargo, para los episodios inferior y superior no disponemos de evidencias que nos permitan aproximar una datación. Por ello, la secuencia de cambios ambientales que se puede derivar del análisis polínico es ante todo una secuencia relativa, comparable en cualquier caso con otros registros polínicos regionales.

Ésta señala dos rasgos especialmente destacados para el episodio de más interés, el central. El primero es el incremento general de la presencia de polen arbóreo, especialmente de pinos, que apunta a la sustitución de un paisaje contextual predominantemente abierto por otro en el que las áreas arboladas ocupan una porción sustancial del espacio. En cualquier caso, esta asunción únicamente puede suponerse en un ámbito regional que no local, ya que en el área inmediata del yacimiento el bosque climácico, el carballal, se reduce como consecuencia de una dinámica antrópica continuada en el tiempo; dinámica ésta que acompaña incluso la desaparición progresiva del matorral mediante el uso del fuego como elemento deforestador.

En principio, esto parece contrastar con la mayor parte de las secuencias polínicas disponibles hasta la fecha para la Edad del Hierro en Galicia (p.e. Díaz Fierros et al. 1979; Aira Rodríguez 1996; Aira Rodríguez et al. 1989; Aira Rodríguez y Saá Otero 1989; Rodríguez López et al. 1993-1994), donde resulta habitual documentar la expansión progresiva de procesos de deforestación. Esta singularidad debe matizarse a partir de cuatro cuestiones:

Primero, la posibilidad de que la única muestra fértil de este episodio introduzca una cierta distorsión en la representatividad real de los resultados. De hecho, tal muestra refleja fielmente lo que ocurre en otros yacimientos gallegos de la misma cronología: deforestación asociada a procesos antrópicos. El hecho de que la única muestra fértil de nuestro estudio arroje porcentajes elevados de cobertura arbórea debe interpretarse únicamente como resultado del progreso de formaciones forestales alejadas, de pinares, que en buena lógica no habrían sufrido los avatares de la antropización producida en el entorno inmediato del castro. Sin embargo, el carballal local, el bosque predominante en el entorno del área estudiada, sí habría continuado deforestándose como consecuencia de la recurrencia de procesos de incendio ya documentados en el episodio inferior.

En este sentido, resulta apropiado señalar aquí la importancia que tiene en los análisis polínicos discernir aquellos palinomorfos que han de interpretarse como elementos de la flora local frente a los que representan la vegetación regional (Janssen 1981); pues en el caso de los pinos, dado su carácter anemófilo, es normal que suelan estar sobrerrepresentados en los espectros polínicos, más cuando la apertura del paisaje, como acontece en el episodio central de Follente, habría facilitado su incorporación a los sedimentos. En la mayor parte de las secuencias polínicas procedentes de zonas montañosas gallegas, en las mismas fechas que en Follente, se asiste a un aumento porcentual de los pinares altimontanos (Ramil Rego 1993). Con más detalle, en la secuencia polínica analizada en el yacimiento de Alto do Castro, fechada antes de finales del siglo $\mathrm{V}$ a.C. (Cobas Fernández y Parcero-Oubiña 2006), en su episodio superior se aprecia una relativa recuperación del polen arbóreo, de la mano especialmente del pino.

Segundo, el hecho de que la mayor parte de los procesos deforestadores más intensos suelen documentarse en contextos cronológicos más tempranos, de inicios de la Edad del Hierro. En yacimientos castreños de la provincia de Pontevedra, como los castros de Penalba (Campolameiro), Torroso (Mós) y Troña (Ponteareas), su análisis polínico (Aira Rodríguez et al. 1989; Aira Rodríguez y Saá Otero 1989) demuestra que la antropización del bosque climácico, el carballal, se produce en los primeros siglos del I milenio a.C. (Penalba, Torroso) o a mediados de éste (Troña). En todos estos casos, como en el episodio inferior, más aún en el central, la progresiva deforestación del carballal va acompañada de la preponderancia de palinomorfos antrópicos y del 
desarrollo de formaciones arbustivas propias de las etapas seriales degradativas del bosque (brezales, tojales, jarales). Aunque sea únicamente como una aproximación relativa, es probable que el episodio inferior de Follente esté representando un período contemporáneo del marco cronológico antes citado.

El hecho de que la cronología del episodio inferior estudiado no pueda ser precisada impide (hecho frecuente en los yacimientos arqueológicos, que se comportan aquí como "ventanas cronológicas cerradas", López Sáez et al. 2003) determinar con precisión la génesis del paleopaisaje del episodio central. En cualquier caso, los espectros polínicos de las muestras del episodio inferior ya dan cuenta de un paisaje cultural abierto y sometido a los influjos de la antropización, por lo que, tal y como antes se dijo, estos datos concuerdan con esos procesos deforestadores documentados en cronologías anteriores a las del episodio central y de alguna manera permiten contextualizar cierta continuidad en la dinámica antrópica entre ambos episodios inferior y central.

Tercero, el hecho de que el incremento del polen arbóreo se produzca esencialmente a costa del de especies arbustivas, mucho más que de las herbáceas, cuyos porcentajes decrecen de forma mucho menor, apunta a una transformación del paisaje menos dramática, y cuyo principal rasgo habría sido antes la degradación del bosque climácico local que la simple expansión de las zonas arboladas.

En cuarto y último lugar resulta necesario reflexionar sobre la circunstancia de la ausencia de polen de cereal precisamente en el episodio central, en estos contextos atribuidos a espacios agrarios, e incluso en la muestra inferior del episodio superior. Los cereales son mayoritariamente especies autógamas, autopolinizantes, y por regla general sus pólenes son excepcionalmente grandes $(>45 \mu \mathrm{m})$, lo que implica baja producción polínica y escasa capacidad dispersiva (López Sáez y López Merino 2005). Ante tales circunstancias, lo más frecuente es que los cereales estén infrarrepresentados en los espectros polínicos, sin que su ausencia haya de implicar imperativamente la inexistencia de cultivos. En este sentido, en el marco de los estudios arqueopaleopalinológicos existe el consenso de confirmar la práctica de actividades agrícolas, en el entorno inmediato de un yacimiento arqueológico, siempre y cuando el porcentaje del morfotipo Cerealia sea al menos de un 3 \% (López Sáez et al. 2003). Cuando se documentan porcentajes más bajos de dicho valor (como ocurre en la muestra 12), o incluso los pólenes de cereales están ausentes en los espectros polínicos, no se pueden atestiguar actividades agrícolas locales pero tampoco se descartan. Puesto que no solamente importa la distancia respecto a las áreas cultivadas, sino también el grado de cobertura de la vegetación herbácea local, la densidad forestal, el método de cosecha y las posibles vías o factores que hayan podido afectar a la dispersión del polen, una presencia aislada de Cerealia no debe significar obligatoriamente la presencia de agricultura en una determinada zona.

En definitiva, la ausencia de polen de cereales en el episodio central del castro de Follente no implica, de manera alguna, que esta zona no fuera cultivada, sino simplemente que por las cuestiones antes señaladas probablemente dicho polen no alcanzó los sedimentos en estudio. De hecho, en el análisis polínico del castro de Penalba el polen de cereal sólo muestra un máximo superior al $3 \%$ a inicios del I milenio a.C. pero tanto antes como después de esta fecha está ausente; en el del castro de Torroso sólo se documenta polen de cereal en dos momentos muy concretos del nivel de ocupación entre 2685-2630 BP; y en cambio en el castro de Troña su presencia es continua entre 2450-2210 BP (Aira Rodríguez et al. 1989; Aira Rodríguez y Saá Otero 1989). En otros yacimientos pontevedreses, como el castro de Cortegada (Cortegada), el polen de cereal está presente de manera discontinua entre los siglos IV a.C. y I d.C. y siempre es inferior al $3 \%$; mientras que en el castro de Montaz (Oleiros) es aún más esporádico entre los siglos V/IV a.C.-I/II d.C.; y en el de Pedra Moura (Gondomar), en cambio, su porcentaje supera con creces el $3 \%$ en el siglo III a.C. (Aira Rodríguez et al. 1989).

La ubicación particular de cada yacimiento y, con ello, de las zonas de cultivos, serían elementos a tener en cuenta a la hora de estudiar las implicaciones tafonómicas sobre la representatividad del polen de cereal en cada uno de ellos y el significado que ha de tener su porcentaje. El caso, quizá más significativo es el de Penalba, donde los estudios polínicos citados muestran una presencia extremadamente puntual de polen de cereal, apenas suficiente para admitir su cultivo local, y en cambio el estudio carpológico (Aira 
Rodríguez et al. 1990) documenta abundantes granos de cereal (Triticum, Panicum), demostrando la existencia, a partir al menos del siglo VI a.C. (aunque probablemente antes, ya que la cronología de ocupación del sitio es algo más antigua), de una importante agricultura cerealista en el yacimiento complementada con la recolección de bellotas. Algo semejante puede suponerse para el castro de Torroso donde también han documentado carporrestos de Triticum y de Hordeum (Dopazo et al. 1996).

En resumen, la presencia de pólenes de cereal en la mayoría de los yacimientos hasta ahora citados, incluyendo entre ellos Follente, hace pensar que en todos ellos se practicó la agricultura del cereal -como básicamente demuestran los estudios carpológicos citados-, aunque dadas las particularidades tanto morfológicas como dispersivas de dicho palinomorfo éste no siempre es documentado polínicamente sin que ello suponga, como se ha visto, la inexistencia de actividades agrícolas.

Igualmente importante es señalar el papel que tuvieron que jugar los incendios de origen antrópico en la conformación de dichos espacios castreños. Hasta fechas relativamente recientes, la diagnosis paleoambiental del fuego se realizaba indirectamente, y siempre de manera subjetiva, a partir de un descenso porcentual de la cobertura arbórea en los espectros polínicos y quizá también por cierta expansión de elementos pirófilos afines a zonas incendiadas caso de Cistus ladanifer o Asphodelus (López Sáez et al. 2003). Sin embargo, muy pocos son los estudios versados en la cuantificación de partículas de microcarbón como elementos definidores de la acción del fuego en contextos arqueológicos, donde la imposibilidad de una reconstrucción diacrónica completa de alguna manera dificulta la interpretación paleoecológica de tal registro. En este sentido, nuestro trabajo aporta la singularidad del estudio de microfósiles no polínicos realmente diagnósticos de la ocurrencia de incendios de origen antrópico en el pasado, en concreto de ascosporas de hongos carbonícolas del género Chaetomium, las cuales podrían venir asociadas a otros palinomorfos relacionados con procesos erosivos (Glomus cf. fasciculatum, Pseudoschizaea circula) derivados tanto de dichos incendios como de la deforestación (López Sáez et al. 1998, 2000). La identificación de Chaetomium en los espectros polínicos de Follente inmediatamente sugiere un papel fun- damental del fuego en la génesis de su paisaje agrario, no sólo en el episodio central sino también antes y a posteriori.

A partir de los resultados de estos análisis, y de su combinación con las evidencias arqueológicas documentadas en el conjunto del entorno del castro de Follente, es posible complementar la propuesta de reconstrucción anterior (Parcero-Oubiña 1999) planteando la secuencia que se observa en la figura 5. Como hemos avanzado, no disponemos de dataciones absolutas directas para estos tres episodios, pero al menos el central, el que más nos interesa, ha proporcionado materiales cerámicos que permiten aproximar con bastante certeza su ubicación. En concreto, destaca la abundancia de cerámica castreña tardía (entre los siglos II a.C. y I d.C., la fase III según la clasificación de Rey Castiñeira 1990-1991, más completo en González Ruibal 2006-2007), que es por otra parte el material más característico de todo el conjunto de estructuras documentadas en el entorno del castro. Adicionalmente, en algunas de ellas (no en la que nos ocupa) se recuperaron en menor medida algunos fragmentos de tegula y de otros elementos de origen romano, que son un porcentaje muy reducido del total de materiales procedentes del entorno del castro (68 fragmentos de ánfora, 5 de Terra Sigillata y fragmentos de 9 recipientes de cerámica común, frente a más de 1000 fragmentos de cerámica de tradición indígena, correspondientes a 129 recipientes, véase el estudio detallado de Isabel Cobas en Parcero-Oubiña 1999). Todo ello nos remite a un momento avanzado dentro del mundo castreño, en parte al menos en época romana, aunque considerando el fuerte peso porcentual de la cerámica de tradición indígena, y la indicación cronológica que aporta el poco material romano adscribible a tipos identificables (ánfora Haltern 70 y de Sigillata gálica Drag. 24/25 (2)), debemos de suponer que las últimas evidencias de ocupación del sitio se sitúan en un momento temprano de la época romana, probablemente no más tarde de la primera mitad del siglo I d.C. Además, conviene destacar que los materiales concretos hallados en la estructura que aquí nos ha ocupado indican, en todo caso, una cronología no más tardía, ya que a la ausencia de materiales romanos documentados

(2) La identificación tipológica del único fragmento de $\mathrm{Si}$ gillata de forma reconocible fue hecha en su día por Catalina López Pérez. 


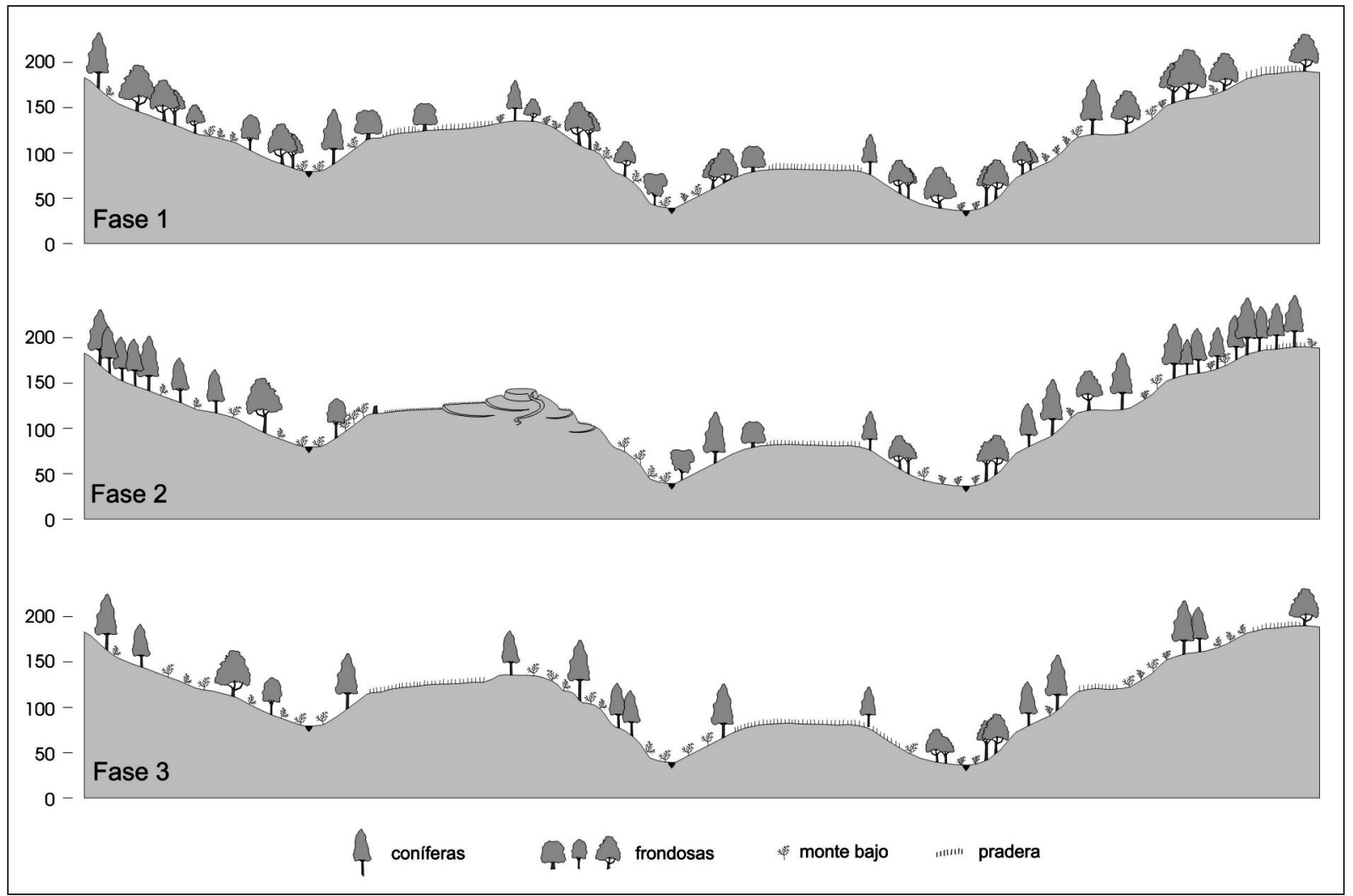

Fig. 5. Reconstrucción de los ambientes vegetales locales, derivados de los resultados del análisis polínico, correspondientes a los tres episodios documentados en la secuencia estratigráfica analizada en el posible espacio agrario del castro de Follente.

aquí se une la presencia de varios fragmentos de borde de un recipiente aristado aparentemente algo más antiguo que el resto de materiales del conjunto (véase el estudio de Cobas Fernández en Parcero-Oubiña 1999: 33 y ss.).

Pese a que las evidencias obtenidas adolecen de una relativa parquedad informativa, debido a la alta proporción de muestras estériles, lo cierto es que el análisis combinado de las evidencias estratigráficas y polínicas nos permite avanzar en la caracterización de las formas de construcción de los paisajes agrarios castreños del noroeste. El castro de Follente resulta un interesante ejemplo de cómo la evidente monumentalización de la acción social en el espacio en este momento no se reduce a la construcción de poblados en posiciones visibles y fuertemente artificializados, sino que se prolonga en la creación de espacios agrarios complejos. Esto se concreta en la convergencia de dos tipos de acciones; por un lado, la movilización activa de trabajo social en la cons- trucción de productos concretos (estructuras de cultivo estables); por otro lado, la alteración progresiva del paisaje a través de los efectos derivados de esas mismas prácticas, como la deforestación de los espacios circundantes, probablemente por medio de procesos amplios de quema que estarían representados por la abundante presencia de carbones en los suelos (una ocurrencia cada vez mejor documentada hacia finales del I milenio a.C. (3)) y que incidirían en caracterizar los importantes procesos de transformación del paisaje acontecidos a partir del surgimiento de la Edad del Hierro y hasta la disolución de las formas de poblamiento fortificado.

(3) Pontevedra-Pombal, X.; Costa-Casais, M.; Ferro, C. y Martínez-Cortizas, A. e.p. "Capítulo 18. Características físicas y químicas de los suelos”. En F. Criado-Boado y A. Martínez Cortizas (eds.): Arte rupestre, paleoambiente y paisaje. Miradas interdisciplinares sobre Campo Lameiro. Trabajos en Arqueología del Paisaje. CSIC. Santiago de Compostela. 


\section{BIBLIOGRAFÍA}

Aira Rodríguez, M.J. 1996: “La vegetación gallega durante la época de ocupación romana a través del estudio del polen fósil". En A. Rodríguez Colmenero (coord.): Lucus Augusti, 1. El amanecer de una ciudad. A Coruña: 25-45.

Aira Rodríguez, M.J.; Ramil Rego, P. y Álvarez Núñez, A. 1990: "Estudio paleocarpológico realizado en el Castro de Penalba (Campolameiro, Pontevedra. España)". Botánica Complutensis 16: 81-89.

Aira Rodríguez, M.J. y Saá Otero, P. 1989: “Contribución al conocimiento de la vegetación holocena (3000-2210 B.P.) de la provincia de Pontevedra a través del análisis polínico". Anales del Jardín Botánico de Madrid 45: 461-474.

Aira Rodríguez, M.J.; Saá Otero, P. y Taboada Castro, T. 1989: Estudios paleobotánicos y edafológicos en yacimientos arqueológicos de Galicia. Arqueoloxía/Investigación 4. Xunta de Galicia. Santiago de Compostela.

Behre, K.E. 1981: "The interpretation of anthropogenic indicators in pollen diagrams". Pollen et Spores 23: 225-245.

Bottema, S. 1975: "The interpretation of pollen spectra from prehistoric settlements (with special attention to liguliflorae)". Palaeohistoria 17: 17-35.

Carrión, J.S. 1992: "Late Quaternary pollen sequence from Carihuela Cave, southeastern Spain". Review of Palaeobotany and Palynology 71: 37-77.

Chouquer, G. y Favory, F. 1991: Les paysages de l'Antiquité. Terres et cadastres de l'Occident romain. Errance. París.

Cobas Fernández, I. y Parcero-Oubiña, C. 2006: Alto do Castro (Cuntis, Pontevedra). Sintesis de resultados y estudio de materiales, campaña 1993. Trabajos en Arqueología del Paisaje 37. CSIC, Instituto de Estudos Galegos Padre Sarmiento. Santiago de Compostela.

Díaz Fierros, F.; Torras Troncoso, M.L. y Vázquez Varela, J.M. 1979: "La evolución de la flora gallega durante el Holoceno". Museo de Pontevedra 33: 3-8.

Dopazo, A.; Fernández, C. y Ramil Rego, P. 1996: "Arqueometría aplicada a yacimientos galaico-romanos del NW peninsular: valoración de la actividad agrícola y ganadera". En P. Ramil Rego, C. Fernández y M. Rodríguez (coords.): Biogeografía Pleistocena-Holocena de la Península Ibérica. Xunta de Galicia. Santiago de Compostela: 317-332.

Girard, M. y Renault-Miskovsky, J. 1969: "Nouvelles techniques de préparation en palynologie appliquées à trois sédiments du Quaternaire final de l'Abri Cornille (Istres, Bouches du Rhône)". Bulle- tin de l'Association française pour l'Etude du Quaternaire 1969 (4): 275-284.

Goeury, C. y Beaulieu, J.L. de 1979: “À propos de la concentration du pollen à l'aide de la liqueur de Thoulet dans les sédiments minéraux". Pollen et Spores 21: 239-251.

González Ruibal, A. 2006-2007: Galaicos. Poder y comunidad en el Noroeste de la Península Ibérica (1200 a.C.-50 d.C.). Brigantium 18-19.

Guilaine, J. (ed.) 1991: Pour une Archéologie Agraire. Armand Colin Editeur. Paris.

Janssen, C. 1981: "Contemporary pollen assemblages from the Vosges (France)". Review of Palaeobotany and Palynology 33: 183-313.

López Sáez, J.A.; López García, P. y Burjachs, F. 2003: "Arqueopalinología: Síntesis Crítica". Polen 12: 5-35.

López Sáez, J.A. y López Merino, L. 2005: “Precisiones metodológicas acerca de los indicios paleopalinológicos de agricultura en la Prehistoria de la Península Ibérica". Portugalia 26: 53-64.

López Sáez, J.A.; van Geel, B.; Farbos-Texier, S. y Diot, M.F. 1998: "Remarques paléoécologiques à propos de quelques palynomorphes non-polliniques provenant de sédiments quaternaires en France". Revue de Paléobiologie 17 (2): 445-459.

López Sáez, J.A.; van Geel, B. y Martín Sánchez, M. 2000: “Aplicación de los microfósiles no polínicos en Palinología Arqueológica”. En V. Oliveira Jorge (coord.): Contributos das Ciências e das Technologias para a Arqueologia da Península Ibérica. Actas 3. ${ }^{\circ}$ Congresso de Arqueologia Peninsular IX (Vila-Real, 1999) 11-20. Porto.

Moore, P.D.; Webb, J.A. y Collinson, M. E. 1991: Pollen analysis. Blackwell. London.

Orejas, A. (coord.). 2006: Arqueología Espacial: Espacios Agrarios. Arqueología Espacial 26. Seminario de Arqueología y Etnología Turolense. Teruel.

Parcero-Oubiña, C. 1999: La Arqueología en la gasificación de Galicia, 7: Hacia una Arqueología Agraria de la Cultura Castreña. Trabajos en Arqueología del Paisaje 9. Grupo de Investigación en Arqueología del Paisaje, Universidade de Santiago. Santiago.

Parcero-Oubiña, C. 2006: "Los paisajes agrarios castreños. Modelos de construcción del espacio agrario a lo largo de la Edad del Hierro del noroeste". Arqueología Espacial 26: 57- 85.

Ramil Rego, P. 1993: "Evolución climática e historia de la vegetación durante el Pleistoceno $\mathrm{Su}-$ perior y el Holoceno en las regiones montañosas del Noroeste Ibérico". En A. Pérez Alberti, L. Guitián Rivera y P. Ramil Rego (eds.): La evolución del paisaje en las montañas del entorno de los Caminos Jacobeos. Xunta de Galicia. A Coruña: $25-60$. 
Reille, M. 1992: Pollen et Spores d' Europe et d' Afrique du Nord. Laboratorie de Botanique Historique et Palynologie. Marseille.

Reille, M. 1995: Pollen et Spores d'Europe et d' Afrique du Nord. Supplement 1. Laboratorie de Botanique Historique et Palynologie. Marseille.

Rey Castiñeira, J. 1990-1991: “Cerámica indígena de los castros costeros de la Galicia occiden- tal: Rías Bajas. Valoración dentro del contexto general de la Cultura Castreña”. Castrelos 3-4: 141-163.

Rodríguez López, C.; Fernández Rodríguez, C. y Ramil Rego, P. 1993-1994: "El aprovechamiento del medio natural en la cultura castreña del noroeste peninsular". Trabalhos de Antropologia e Etnologia 33 (1-2): 285-305. 